\title{
Mobilfunk und Gesundheit
}

Das breite Spektrum der Meinungen zu den gesundheitlichen Auswirkungen der Mobilfunktechnologie wird in diesem Artikel dargelegt und aus der Sicht einer Arbeitsgruppe der Ärztinnen und Ärzte für Umweltschutz bewertet. Die Notwendigkeit vorsorglicher Massnahmen und kontinuierlicher Forschung wird begründet. Bis zur weiteren wissenschaftlichen Klärung der Situation soll vom Ausbau der Mobilfunktechnologie abgesehen werden. Praktizierende Kolleginnen und Kollegen werden in einem wissenschaftlich begleiteten Projekt eine unabhängige medizinische Abklärungs- und Beratungsstelle für betroffene Patientinnen und Patienten aufbauen.

Arbeitsgruppe «Elektromagnetische Felder und Gesundheit» der Ärztinnen und Ärzte für Umweltschutz*

\footnotetext{
Dr. med. Edith Steiner-Rüedi, Dr. med. Yvonne Gilli, med. pract. Cornelia Semadeni, Dr. med. dent Bruno Germann,
} Dr. med. Bernhard Aufdereggen.

Korrespondenz: Dr. med. Edith Steiner-Rüedi Friedbergstrasse 32 CH-8200 Schaffhausen
In den letzten Wochen wurde die Auseinandersetzung um die Frage nach dem Einfluss von Mobilfunkstrahlung auf die menschliche Gesundheit wieder intensiver geführt. Es scheint auch klarer zu werden, dass in dieser Auseinandersetzung um die öffentliche und damit politische Meinung die Ärzteschaft eine nicht unwichtige Rolle spielt.

Das Spektrum der Meinungen ist breit:

- Die mobilfunkindustrienahen «Experten» wollen die Diskussion als beendet erklären und glauben genügend Evidenz zu sehen, um eine Unbedenklichkeitserklärung gegenüber den gesundheitlichen Auswirkungen von hochfrequenter elektromagnetischer Strahlung im Einsatzbereich der Mobilfunktechnologie zu formulieren.

- Im Widerspruch dazu artikulieren sich betroffene Menschen, die ihr Wohlbefinden und ihre Gesundheit durch die negativen Auswirkungen des Mobilfunks massiv betroffen und bedroht sehen. Sie haben sich zusammengeschlossen in BürgerInnen-Bewegungen.

- Die Behörden - das Bundesamt für Umwelt haben ein inzwischen vom Bundesrat beschlossenes Nationales Forschungsprogramm vorgeschlagen, um die Zusammenhänge zwischen elektromagnetischen Feldern und Gesundheit näher untersuchen zu lassen.

- Forscherinnen und Forscher erzielen oft nicht eindeutige Ergebnisse, viele Fragen werden aufgeworfen und auf weiteren Forschungsbedarf wird hingewiesen.

\section{Téléphonie mobile et santé}

Un groupe de travail des médecins en faveur de l'environnement présente dans cet article les multiples avis concernant les effets sur la santé de la téléphonie mobile. Le groupe fournit des arguments quant à la nécessité de prendre des mesures préventives et de continuer les recherches à ce sujet. Jusqu'à l'obtention de données scientifiques plus approfondies, il serait bon de renoncer à tout développement de la technologie mobile. Des confrères en pratique privée vont mettre sur pied, dans le cadre d'un projet bénéficiant d'un accompagnement scientifique, un service $d$ 'investigation et de consultation médicale indépendant pour les patients concernés.

\section{Ist Mobilfunk ein Gesundheitsrisiko?}

Nein! Sagt die Mobilfunkindustrie mit ihren Experten

Unter dem Titel «Mobilfunk - ein Gesundheitsrisiko?» richtete sich das Forum Mobil im Oktober 2006 an uns Ärztinnen und Ärzte der Schweiz. Das Forum Mobil ist ein PR-Instrument der Mobilfunkanbieterindustrie, das sich schon 
verschiedentlich mit entsprechend gewichteten Informationen an die Ärzteschaft gerichtet hat. Die in der Aussendung vermittelte Botschaft geht davon aus, dass sich in letzter Zeit Berichte gehäuft hätten, «wonach von immer mehr Ärzten eine Zunahme von Erkrankungen beobachtet werde, die durch Mobilfunksendestationen verursacht sein sollen.» Aus der Sicht des Forums Mobil präsentiert uns die Wissenschaft ein ganz anderes Bild. Wir Ärztinnen und Ärzte sollen davon überzeugt werden, dass «die grosse Mehrzahl der Studien keinen Zusammenhang zwischen Funkwellen und negativen Einflüssen auf die Gesundheit zeigt».

Einen Schritt weiter geht das vom Forum Mobil initiierte Projekt MMG: Medizin - Mobilfunk - Gesundheit. Als Ausganglage für dieses Projekt wird vom beauftragten PR-Büro angeführt, dass «basierend auf dem heutigen Wissensstand keine gesundheitliche Gefährdung durch elektromagnetische Felder besteht, solange die gültigen Grenzwerte und Empfehlungen eingehalten werden». Trotzdem würden «sich viele Leute Sorgen um die eigene Gesundheit machen, speziell in Bezug auf die Strahlung von Handybasisstationen und Handys selbst». Als ein Teil des Problems wird die allzu vorsichtige Formulierung der «Wissenschaft» angeführt. Aber das wesentliche Problem scheinen wir Ärztinnen und Ärzte zu sein: «Unterstützt wird diese diffuse Angst aber auch durch das Verhalten vieler Ärzte. Umfragen haben nämlich gezeigt, dass eine Mehrheit der Allgemeinpraktiker in der Schweiz der Meinung ist, Mobilfunkstrahlung sei für konkrete Beschwerden in der Bevölkerung verantwortlich. Offenbar werden die bis heute verfügbaren Resultate aus Studien und Beobachtungen nicht in die Überlegungen übernommen oder erst gar nicht wahrgenommen.» Ein PRBüro hat «Experten» zu einer Sitzung eingeladen. Diese sollen ein Konsenspapier absegnen und Empfehlungen an den Hausarzt formulieren. Geplant sind auch Patientenbroschüren.

Dass die Mobilfunkindustrie auch im Forschungsbereich Einfluss ausübt, zeigte eine Studie der Institute für Sozial- und Präventivmedizin der Universitäten Bern, Basel und Bristol [1]: Nur 30\% derjenigen Mobilfunkstudien, welche von der Industrie finanziert wurden, zeigten gesundheitsrelevante Effekte, während über 70\% der gemischt oder öffentlich finanzierten Studien einen Effekt zeigen.

Die WHO hat in den letzten Jahren unter der Leitung von Dr. Mike Repacholi ein EMF-Projekt vorangetrieben und Positionspapiere (Fact-
Sheets) publiziert. Diese haben die Gesundheitsgefahren durch den Einfluss von elektromagnetischen Feldern oft als sehr gering bewertet. Dr. Mike Repacholi ist von Kritikern wiederholt die Nähe zur Industrie vorgehalten worden. Nachdenklich stimmt in diesem Zusammenhang die Tatsache, dass Dr. Repacholi nur wenige Monate nach dem Verlassen seines Postens bei der WHO als Berater der Elektroindustrie (Connecticut Light and Power Company) aufgetreten ist [2].

\section{Ist Mobilfunk ein Gesundheitsrisiko?}

\section{Wir wissen noch zu wenig, um die Frage klar zu beantworten - sagen viele Wissen- schaftlerinnen und Wissenschaftler}

Die Ärztinnen und Ärzte für Umweltschutz verfolgen seit Jahren in engem Austausch mit Wissenschaftlern den aktuellen Wissensstand.

Die Frage, ob elektromagnetische Felder bzw. nicht ionisierende Strahlen (NIS) sich negativ auf die Gesundheit auswirken können, wird von den Wissenschaftlern kontrovers beantwortet. Der Erkenntnisprozess, ob und in welchem Ausmass elektromagnetische Felder unter Alltagsexposition krank machen können, braucht Zeit. Die Datenlage ist noch unzureichend. Die Studienergebnisse sind zum Teil widersprüchlich und die technologische Entwicklung ist derart rasant, dass die Prüfung des Gesundheitsschutzes dem technischen Fortschritt nachhinkt. Auf jeden Fall wird auf den Bedarf nach weiterer Forschung hingewiesen [3, 4].

Forscher weisen darauf hin, dass gesundheitliche Probleme durch die Exposition mit hochfrequenten elektromagnetischen Feldern entstehen können, mahnen zur Vorsicht und zu mehr wissenschaftlicher Arbeit. So etwa weist Prof. Primo Schär von der Universität Basel anlässlich seiner Präsentation in der von der Forschungsstiftung Mobilkommunikation organisierten Veranstaltung «Science Brunch» in Zürich im Juni 2006 in einer Folie zusammenfassend darauf hin [5]:

- «Wir wissen, dass elektromagnetische Felder unsere Gene stressen!

- Wir wissen nicht, ob dieser Stress die Gene nachhaltig schädigen kann!

- Es ist wahrscheinlich, dass sich die gentoxische Wirkung der EMF nicht auf alle Individuen gleichermassen auswirkt!

- Weniger ist mehr?

- Handys mit niederen 〈SAR>-Werten;

- Zeit der Exposition limitieren.

- Mehr Forschung!» 


\section{Ist Mobilfunk ein Gesundheitsrisiko?}

Die Behörden halten an den geltenden Grenzwerten fest, sehen aber Handlungsbedarf im Forschungssektor

Auf Initiative des Bundesamtes für Umwelt (BAFU) und mit Unterstützung weiter Kreise in Verwaltung, Politik, Forschung und von Nichtregierungsorganisationen wurde vom Bundesrat im März 2006 das Nationale Forschungsprogramm (NFP 57) «NIS, Gesundheit und Umwelt» angenommen. Für dieses Projekt wurden 5 Millionen Schweizer Franken bewilligt. Unabhängig hier staatlich - finanzierte Forschung hat in diesem umstrittenen Forschungsgebiet eine eminente Bedeutung. So ist sie der Einflussnahme der Mobilfunkindustrie entzogen.

Im Bericht einer nationalen interdepartementalen Arbeitsgruppe zum Gesundheitsschutz vor nichtionisierender Strahlung wird im April 2006 festgehalten, dass das Nationale Forschungsprogramm einen grossen Schritt in die richtige Richtung darstellt. Die Arbeitsgruppe fordert jedoch noch mehr: «Es sind die strukturellen und finanziellen Voraussetzungen für eine langfristig angelegte Forschung zu den Gesundheitsrisiken von nichtionisierender Strahlung zu schaffen. Es ist ein nationales Krebsregister einzurichten.» [6]

\section{Ist Mobilfunk ein Gesundheitsrisiko?}

Die Ärztinnen und Ärzte für Umweltschutz (AefU) halten im Interesse der betroffenen Personen an einem Moratorium für den Weiterausbau der Mobilfunktechnologie fest In unserer Sprechstunde sehen wir von der AefU, wie auch - wenn wir der oben angeführten Umfrage von Huss et al. Glauben schenken wollen viele Kolleginnen und Kollegen, in der Praxis Menschen, die plausibel Beschwerden im Zusammenhang mit der Mobilfunktechnologie (Antennen und/oder Handys und/oder DectTelefonen) schildern. Die zahlreich dokumentierten Einzelfallberichte mit Beschwerden um Basisstationen oder im Zusammenhang mit hausgemachter Mobilfunkbelastung konnten bisher epidemiologisch nicht sicher in einen Zusammenhang gebracht werden. Eine wissenschaftlich gesicherte Zuordnung der Beschwerden zu den Quellen gelang nicht eindeutig. Eine erste Studie aus Österreich, die die gemessene Belastung der Menschen in ihren Schlafräumen mit Symptomen in einen Zusammenhang brachte, ergab einen Zusammenhang zwischen der Höhe der Strahlenbelastung und den Beschwerden [7].
Daher ist vor dem weiteren Ausbau der Mobilfunktechnologie die Frage der Beeinflussung des Wohlbefindens von empfindlichen Menschen zu klären.

Wir erhoffen uns vom NFP 57 in diesem Bereich eine zusätzliche Klärung der Situation. Weiterhelfen wird sicher auch die neue Möglichkeit der individuellen Dosimetrie, die bis vor kurzem nicht zur Verfügung stand.

Über die offene Frage der Beeinflussung des menschlichen Genoms durch die Mobilfunkstrahlung wurde weiter oben berichtet. Auch sind die Fragen rund um den Einfluss von Mobilfunkstrahlung auf Hirntumore nicht definitiv geklärt. Hardell berichtet in einer aktuellen Übersichtsarbeit über vermehrtes Auftreten von Akustikusneurinomen und andern Hirntumoren bei Langzeitnutzung von Mobilfunktechnologie [8]. Aufhorchen lassen auch die aktuellen Resultate eines europäischen Forscherteams, das eine fast 40\%ige Zunahme von Gliomen bei langjährigen Benutzern von Mobiltelefonen (10 Jahre und mehr) auf der Telefonbenutzungsseite feststellte [9]. Die Beeinflussung des menschlichen Genoms durch die Mobilfunkstrahlung käme hier als mögliches Modell in Frage. Um die Zusammenhänge zwischen Krebsentstehung und Mobilfunk genauer abzuklären, unterstützen wir die Forderung der interdepartementalen Arbeitsgruppe nach kontinuierlicher und unabhängig finanzierter Forschung und nach einem nationalen Krebsregister. Im Elektrosmogfenster unserer Homepage (www.aefu.ch) finden Sie eine ausführliche Übersicht über den erfahrungsmedizinischen und naturwissenschaftlichen Erkenntnisstand.

\section{Ist Mobilfunk ein Gesundheitsrisiko?}

\section{Wo Bedenken für eine Beeinflussung der Gesundheit der Menschen bestehen, ist Vorsorge angebracht}

Es ist Aufgabe der Ärzteschaft, nicht nur zur Heilung von Krankheiten beizutragen, sondern auch bei einem potentiellen Gesundheitsrisiko zur Vorsorge zu mahnen.

Aufgrund des jetzigen Wissensstandes unterstützen wir deshalb die Forderung der Bundesbehörden nach einem vorsorgeorientierten Umgang mit nicht ionisierenden Strahlen im Alltag [10], insbesondere begrüssen wir Bestrebungen nach vermehrter Aufklärung der Bevölkerung hinsichtlich des Gefahrenpotentials von NISemittierenden Geräten und entsprechenden Empfehlungen zur Reduktion der Strahlenbelastung. Auf unserer Homepage [11] finden 
Sie einen Internetratgeber für Ihre Patienten, mit einfachen und kostenlosen Tips zur individuellen Reduktion der Elektrosmogbelastung.

\section{Unabhängige umweltmedizinische Beratungsstelle}

Wir wollen mit der unabhängigen umweltmedizinischen Beratungsstelle dazu beitragen, dass betroffene Menschen kompetent und unabhängig beraten und betreut werden

Eine 2005 in der Schweiz durchgeführte Bevölkerungsbefragung ergab, dass 5\% der Bevölkerung Beschwerden im Zusammenhang mit Elektrosmog angaben, wobei nur 13\% der Betroffenen deshalb ihren Hausarzt aufsuchten. In einer Ärztebefragung aus dem Jahr 2005 gaben Kolleginnen und Kollegen aus der Grundversorgung an, dass bei $2 / 3$ von ihnen in Konsultationen schon mal Beschwerden im Zusammenhang mit elektromagnetischen Feldern zur Sprache gekommen sind. Dabei sind Arztkonsultationen wegen Problemen rund um Elektrosmog selten, aber zunehmend. Interessant ist aber, dass 54\% der Schweizer Ärztinnen und Ärzte einen Zusammenhang zwischen den geschilderten Beschwerden und der vermuteten Elektrosmogquelle als plausibel erachteten.

Die Diskrepanz zwischen Erfahrungsmedizin und Wissenschaft erfordert eine unvoreingenommene, unabhängige und möglichst sachliche Haltung der Ärzteschaft. Eine Pilotstudie einer umweltmedizinischen Beratungsstelle unter der Leitung von Prof. Dr. med. Charlotte Braun vom Institut für Sozial- und Präventivmedizin Basel aus dem Jahr 2001 zeigte, dass auch ein bestens informiertes interdisziplinäres Expertenteam bei einem Teil der untersuchten elektrosensiblen Patienten Beschwerden im Zusammenhang mit Elektrosmog als plausibel beurteilte, obwohl die geltenden Grenzwerte eingehalten worden waren.

Unter diesen Aspekten begründet sich unser Vorhaben, eine unabhängige umweltmedizinische Beratungsstelle zu schaffen. Ein loses Netzwerk umweltmedizinisch geschulter Grundversorger soll Betroffenen und Ärztinnen und Ärzten eine niederschwellige und unabhängige
Anlaufstelle bieten. Ein modulartig aufgebautes standardisiertes Vorgehen mit bedarfsweisem Beizug von Spezialärzten/Universitären Instituten und Baubiologen bzw. Messtechnikern soll eine wissenschaftliche Aufarbeitung der ermittelten Daten ermöglichen. So soll eine Schnittstelle zwischen Erfahrungsmedizin und Grundlagenforschung geschaffen werden. Wir werden darüber berichten.

\section{Literatur}

1 Huss A, Egger M, et al. Source of funding and results of studies of health effects of mobile phone use: systematic review of experimental studies. Environ Health Perspect. 2006.

2 Microwave News. Nov 13, 2006 und Antwort von Repacholi vom 15. November 2006.

3 Schreier N, Huss A, Roosli M. The prevalence of symptoms attributed to electromagnetic field exposure: a cross sectional representative survey in Switzerland. Soz Praventivemed. 2006;51(4):202-9

4 Pearce MS, Hammal DM, Dorak MT, McNally RJ, Parker L. Paternal occupational exposure to electro-magnetic fields as a risk factor for child and young adults: a case-control study from the North of England. Pediatr Blood Cancer. 2006(epub).

5 Der Vortrag von Prof. Primo Schär kann eingesehen werden unter www.mobile-research.ethz.ch/ dienstleistungen (unter Science Brunch 4, Referat Primo Schär).

6 Nichtionisierende Strahlung und Gesundheitsschutz in der Schweiz; Überblick, Handlungsbedarf und Empfehlungen. Bericht einer interdepartementalen Arbeitsgruppe des Bundes in Erfüllung des Postulats Sommaruga. April 2006.

7 Hutter HP, Moshammer H, Wallner P, Kundi M. Subjective symptoms, sleeping problems, and cognitive performance in subjects living near mobile phone base stations. Occup Environ Med. 2006;63(5):307-13.

8 Hardell L, Mild KH, Carlberg M, Soderqvist F. Tumour risk associated with use of cellular telephones or cordless desktop telephones. World J Surg Oncol. 2006;4:74.

9 Lahkola A, Anvinen A, Raitanen J, Schoemaker MJ, Christensen HC, Feychting M, et al. Mobile phone use and risk of glioma in 5 North European countries. Int J Cancer. 2007 (epub).

10 Einzusehen unter www.bag.admin.ch unter dem Thema «Strahlung».

11 www.aefu.ch. 